8 Bebbington P. Childhood sexual abuse and psychosis: aetiology and mechanism. Epidemiol Psichiatr Soc 2009; 18: 284-93.

9 Read J, Perry B, Moskowitz A, Connolly J. The contribution of early traumatic events to schizophrenia in some patients: a traumagenic neurodevelopmental model. Psychiatry 2001; 64: 319-45.

10 Bentall RP, Fernyhough C. Social predictors of psychotic experiences: specificity and psychological mechanisms. Schizophr Bull 2008; 34: 1012-20.
11 Read J, Hammersley P, Rudegeair T. Why, when and how to ask about child abuse. Adv Psychiatr Treat 2007; 13: 101-10.

12 NHS Confederation. Briefing 162: Implementing National Policy on Violence and Abuse. Ministry of Health, 2008.

13 Albee GW. Revolutions and counter-revolutions in prevention. Am Psychol 1996; 51: $1130-3$

\section{psychiatry} in pictures

\title{
Ashanti fertility dolls (Akua'ba)
}

\section{Malcolm P. Weller}

'Belief in myths allows the comfort of opinion without the discomfort of thought.' John F. Kennedy

In superstitions, intuitive concepts and spurious attribution coexist with acquired rational knowledge. In animals 'superstitious learning' based on intermittent rewards, unlike the withdrawal of predictable reward, is difficult to extinguish.

It might be thought that increasing environmental control would reduce reliance on unverified beliefs. Nevertheless, despite the conflict with religious prohibitions, in American society approximately one quarter believe in astrology, clairvoyance, ghosts and communication with the dead. Such beliefs, and good-luck charms, are often important parts of people's lives.

Akua'ba (from Akua, a day-name for a female born on a Wednesday, and ba, child; hence, Akua's child) refers to the fertility doll carved from wood by the Ashanti (more correctly, Asante), a major ethnic group of the Ashanti Region of Ghana. At their height they dominated most of Ghana, as well as parts of Togo and the Ivory Coast.

Fertility dolls are recommended by a herbalist, or generally a priest, and the woodcarver has high status, reinforcing prevailing belief. Like normal children, they are dressed and tied to the back, or form part of a home shrine when not being carried.

The line of descent in Ashanti culture is matrilineal. Dolls are thought to represent an ideal of feminine beauty, the likelihood of having a beautiful female child being increased in those who carry the doll. Accordingly, the dolls were also carried by pregnant women, but more often by infertile women. Apart from the normal desire for motherhood, infertility could raise suspicions of witchcraft. Because of the premium on fertility and the stress of infertility, associated physiological perturbations might be thought to aggravate infertility.

Anecdotal evidence suggests that women's fertility is lower in stressful circumstances and that conception is more frequent during or after a holiday, or after adoption, following a protracted period of infertility. The idea that stress limits fertility would be a natural barrier to population expansion at times of drought and famine and would be a mechanism for balancing population to resources. In support of these ideas, extreme weight loss, as in anorexia nervosa, leads to amenorrhea. Ovulation in the Kung! of the Northern Kalahari desert area of Botswana, a non-contraceptive using population with a low fertility and a birth space interval of greater than 3 years, is linked to the rains, but otherwise there is no evidence to support these suppositions in humans when objective data are rigorously analysed, even in artificial fertility treatment.

It is estimated that one in three or four Ashanti women possessed a doll. Ironically, fertility in Ghana is low compared with most other African countries. The elevation of anecdote over evidence might be summarised in the present example by saying that the wish to mother is the thought.

With grateful thanks to Adrian Bird for valuable input and helpful discussion.

References available on request to Professor Malcolm P. Weller, School of Health and Social Sciences, Middlesex University, email: psychiatry@weller.tv. Image (c) Skeptiseum. Reproduced with permission. 